\title{
Endoscopic clipping prior to GVO vs. GVO in IGV1 bleeding: a multicenter retrospective clinical trial $\square$
}

\section{다(1) $\odot \Theta$}

\author{
Authors \\ Huixian $\mathrm{Li}^{1}$, Dong $\mathrm{Ye}^{2}$, Ping $\mathrm{Li}^{3}$, Derun Kong ${ }^{1}$ \\ Institutions \\ 1 Department of Gastroenterology, Anhui Geriatric \\ Institute, The First Affiliated Hospital of Anhui Medical \\ University, Anhui, China \\ 2 Department of Infectious Diseases, Infectious Diseases \\ Hospital of Handan City, Hebei, China \\ 3 Department of Gastroenterology, Ditan Hospital \\ affiliated of Capital Medical University, Beijing, China
}

submitted 13.10.2018

accepted after revision 14.2.2019

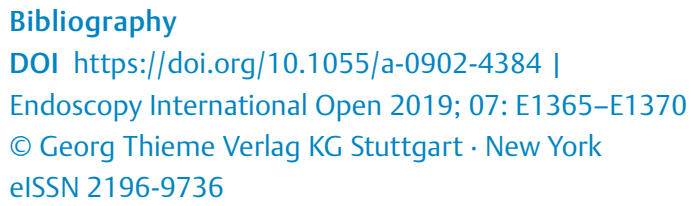

Corresponding author

Derun Kong, MD, Department of Gastroenterology, Anhui Geriatric Institute, First Affiliated Hospital of Anhui Medical University, Jixi Road 218, Hefei 230022, China

Fax: +86-551-63633742

kongderun168@163.com

\begin{abstract}
Background and study aims Bleeding from isolated gastric varices type I (IGV1) is more dangerous and fatal than other types of esophageal variceal hemorrhages. However, optimum treatment for bleeding from IGV1 remains undefined. This retrospective study compared the efficacy of endoscopic clipping prior to N-butyl-2-cyanoacrylate injection (GVO) and only GVO for treatment of IGV1.
\end{abstract}

Patients and methods Data were collected retrospectively at three medical centers. Ninety-six patients were enrolled between March 2015 and April 2017 and divided into two groups: group I (patients with endoscopic clipping prior to GVO, $n=46$ ) and group II (patients with only GVO, $n=50)$. Computed tomography angiography (CTA) was performed to evaluate the status of gastrorenal shunts (GRS). Results GRS was found in 59 of 66 patients (59/66). The groups did not differ in baseline characteristics. Initial hemostasis was successfully achieved in all patients. There was a significant difference in volume of N-butyl-2-cyanoacrylate administered $(3.39 \pm 1.20 \mathrm{~mL}$ in group I versus 2.53 $\pm 1.05 \mathrm{~mL}$ in group $\|(P=0.03)$. Rebleeding occurred in $4.35 \%$ of patients in group I and $18.00 \%$ in group II ( $P=$ $0.007)$. Variceal obliteration was achieved in all patients in group I and $72 \%$ in group II $(P<0.001)$ with only one session. Rate of complications was similar in both the groups with the exception of one patient in group II who developed ectopic cerebral embolism.

Conclusions Endoscopic clipping prior to GVO may be an appropriate alternative to GVO as a treatment modality for IGV1 bleeding.

\section{Introduction}

Esophageal and gastric variceal bleeding is a severe complication in cirrhotic patients with portal hypertension. Although bleeding from gastric varices (GV) occurs less frequently than from esophageal varices (EV), GV are associated with severe blood loss, more transfusions, higher rebleeding and mortality $[1,2]$. Among the different treatment modalities available for GV bleeding, endoscopic therapy is considered optimum treatment as N-butyl-2-cyanoacrylate injections (GVO) can rapidly block variceal hemorrhages. The widely adopted Sarin classifi- cation for GV appropriately differentiates gastroesophageal junctional varices (GOV1, GOV2) from isolated gastric varices (IGV1, IGV2) [3]. Compared to endoscopic variceal ligation, endoscopic variceal obturation with tissue adhesive such as $\mathrm{N}$ butyl-2-cyanoacrylate is a more effective treatment for IGV1 with better control of hemorrhage as well as lower rates of rebleeding. However, its use is associated with a high risk of systemic ectopic embolization, especially in GV patients with gastrorenal shunts (GRS) or splenorenal shunts (SRS) [4, 5].

To prevent risk of ectopic embolism, we utilized titanium clips to clip large gastric varices prior to N-butyl-2-cyanoacry- 
late injection. The current study was a multicenter retrospective clinical trial to test the efficacy and safety of endoscopic clipping prior to GVO versus traditional GVO in treatment of IGV1 bleeding.

\section{Patients and methods}

\section{Patients}

This retrospective clinical trial was conducted at three medical centers in China: The First Affiliated Hospital of Anhui Medical University, Beijing Ditan Hospital and Infectious Diseases Hospital of Handan City. From March 2015 to April 2017, cirrhosis patients with a history of upper gastrointestinal bleeding and endoscopic presentation of IGV1 were enrolled. Patients with 1) previous history of sclerotherapy, endoscopic band ligation, portosystemic shunt surgery or interventional therapy; and 2) hepatocellular carcinoma, other malignancy and debilitating diseases were excluded from the study.

Of 96 patients included in the study, 46 underwent endoscopic clipping prior to GVO (group I), and 50 underwent only GVO (group II). CTA examination was performed to evaluate the relationship between GV and GRS/SRS. Severity of cirrhosis was based on the Child-Pugh classification. Variceal forms were endoscopically graded as: F1 (linear small varices), F2 (beaded medium-sized varices), and F3 (nodular or tumorous large varices) [6].

This study was approved by the ethics committee of all the hospitals and informed consent was gathered from each patient prior to study inclusion.

\section{Endoscopic procedures}

Before therapeutic endoscopy, patients received vasoactive drugs (somatostatin or terlipressin) as well as prophylactic antibiotics [7].

Endoscopic clipping prior to GVO was performed as follow: Any large amount of blood in the stomach was removed to facilitate clear endoscopic view, titanium clips (Instinct; Cook Medical, Bloomington, Indiana, United States) were then deployed on the varices. Generally, the afferent branch of the varix was clipped first followed by the efferent branch of the varix. Any large intravenous cavity on the upper part of the vein was clipped to reduce the volume of cyanoacrylate required. Overall, determination of the correct position of the clips was based on size and the number of gastric varices was assessed endoscopically. A “modified Sandwich method" (lauromacrogol, $\mathrm{N}$-butyl-2-cyanoacrylate, and sodium morrhuate) was used to complete the obturation. First, $4 \mathrm{~mL}$ of lauromacrogol (Tianyu Pharmaceutical Co. Ltd., Shanxi, China) was injected into a gastric varix followed by N-butyl-2-cyanoacrylate (Histoacryl; B. Braun, Melsungen, Germany) injection into the same varix. Finally, $2 \mathrm{~mL}$ of normal saline was rapidly injected to flush the glue into the varices. N-Butyl-2-cyanoacrylate dosage was determined by the diameter of gastric varices, usually 1 -cm diameter varices should be injected with 1-mL tissue gel, more than $1 \mathrm{~cm}$ in accordance with $1 \mathrm{~mL} / \mathrm{cm}$ supplementary dosage. The first injection site was selected between two clips. Additional glue was injected on each side of the clip until the whole varix had been solidified. However, clip deployment could cause the vessel to be broken and a little blood to ooze out. Any bleeding was immediately blocked by injecting the same mixture. Thereafter, the injection needle was immediately withdrawn and the needle sheath was used to slightly compress the varices for a few seconds to prevent $\mathrm{N}$-butyl-2-cyanoacrylate from flowing out $\triangleright$ Video 1. GVO in group II was performed following the exact protocol mentioned above.

Post-treatment, patients were given $40 \mathrm{mg}$ omeprazole intravenously every 12 hours for 2 days and then $20 \mathrm{mg}$ orally twice a day for 2 weeks.

\section{Follow-up and clinical assessment}

Endoscopy and CTA were done the fourth week after surgery. Endoscopic and GVO treatment were repeated until complete eradication of variceal. Subsequently, follow-up endoscopy was performed every 3 months, which was extended to every 6 months if there were no visible gastric varices.

The study end points were: 1 ) rate of rebleeding originating from gastric varices within the first 72 hours after endoscopic treatment; 2) rate of complications; 3 ) median survival time after procedure; and 4) post-procedure mortality.

Successful hemostasis was defined as no active bleeding from gastric varices within 72 hours. Rebleeding was defined as new onset of hematemesis, melena, or hematochezia with variceal bleeding within 72 hours of stable vital signs after endoscopic management. Variceal obliteration was considered when varices reduced to F1 or non-visualization of patent GV. Complications were defined as any special event that required active treatment or prolonged hospitalization. Treatment failure was defined as two or more rebleeding events from varices or death.

\section{Statistical analysis}

Quantitative data were represented as mean \pm standard deviation (SD). Significant differences between the only GVO control and clipping prior to GVO treated groups were determined by two-sample Student's $t$ test. Comparison of categorical data (n [\%]) was done using Chi square test. All analyses were conducted using SPSS 16.0 software. $P<0.05$ was considered to be statistically significant.

\section{Results}

Both groups had similar demographics, major laboratory index, Child-pugh grade, and follow-up period ( $\downarrow$ Table 1 ).

CTA examination in 46 patients in group I and 20 patients in group II revealed 59 patients (59/66) had GRS with diameters of $1.20 \pm 0.20$ (range, $0.60-1.50$ ) cm. At 1 month-follow-up, CTA showed well blocked GV and GRS in 32 patients in group I. Mean number of clips used per session in group I was $2.62 \pm 1.17$ (range, $1-5$ ). Mean number of injection points per session and volume of $\mathrm{N}$-butyl-2-cyanoacrylate were higher in group I compared with group II $(4.17 \pm 0.30$ vs. $3.39 \pm 1.20$ points; $3.39 \pm$ 1.20 vs. $2.53 \pm 1.05 \mathrm{~mL} ; P<0.05)$.

Initial hemostasis was successful in all patients. In group I, two patients had melena and hematochezia 5 and 16 days, 
- Table 1 Characteristics of the study group.

\begin{tabular}{|c|c|c|c|}
\hline Variable & Group I $(n=46)$ & Group II $(n=50)$ & $P$ \\
\hline Age (years, mean $\pm S D$ ) & $50.30 \pm 11.75$ & $52.19 \pm 8.17$ & NS \\
\hline Male/female & $30 / 16$ & $35 / 15$ & NS \\
\hline Etiology (viral/alcohol/other) & $31 / 11 / 4$ & $30 / 13 / 7$ & NS \\
\hline Child-Pugh class $(\mathrm{A} / \mathrm{B} / \mathrm{C})$ & $14 / 30 / 2$ & $15 / 31 / 4$ & NS \\
\hline Serum albumin (g/L) & $30.57 \pm 6.20$ & $32.14 \pm 6.14$ & NS \\
\hline Serum bilirubin (umol/L) & $23.62 \pm 12.87$ & $19.91 \pm 9.84$ & NS \\
\hline Ascites present & $21(46 \%)$ & $26(52 \%)$ & NS \\
\hline Size of gastric varices (F1/F2/F3) & $0 / 5 / 41$ & $0 / 7 / 43$ & NS \\
\hline Follow-up time (months) & $10.47 \pm 6.04$ & $10.47 \pm 6.04$ & NS \\
\hline \multicolumn{4}{|c|}{ NS, not significant, $P>0.05$; values expressed as mean \pm SD. } \\
\hline Variable & Group I $(n=46)$ & Group II $(n=50)$ & $P$ \\
\hline Initial hemostasis & $46(100 \%)$ & $50(100 \%)$ & NS \\
\hline Dose of N-butyl-2-cyanoacrylate (mL) & $3.39 \pm 1.20$ & $2.53 \pm 1.05$ & .03 \\
\hline Injection points per session & $4.17 \pm 0.30$ & $3.39 \pm 1.20$ & .02 \\
\hline Rebleeding & $2(4.35 \%)$ & $9(18 \%)$ & .007 \\
\hline \multicolumn{4}{|l|}{ Variceal obliteration } \\
\hline After one session & $46(100 \%)$ & $36(72 \%)$ & $<.001$ \\
\hline After two sessions & & $50(100 \%)$ & \\
\hline \multicolumn{4}{|l|}{ Complications } \\
\hline Fever & $10(21.7 \%)$ & $8(16 \%)$ & NS \\
\hline Abdominal pain & $6(13 \%)$ & $7(14 \%)$ & NS \\
\hline Bleeding from post-treatment variceal ulcer & 0 & $5(10 \%)$ & NS \\
\hline Spontaneous bacterial peritonitis & 0 & $2(4 \%)$ & NS \\
\hline Hepatic encephalopathy & $2(4.3 \%)$ & $3(6 \%)$ & NS \\
\hline Ectopic embolism & 0 & $1(2 \%)$ & NS \\
\hline Mortality & $3(6.5 \%)$ & $3(6 \%)$ & NS \\
\hline
\end{tabular}

respectively, after endoscopy, the rebleeding rate in group I was $4.35 \%$ (2/46). In the traditional N-butyl-2-cyanoacrylate treatment group, nine patients had postoperative hematemesis or melena. Rates of rebleeding and time to rebleed in group II were $18.00 \%(9 / 50)$ and $11.78 \pm 8.02$ (range, 4-28) days, respectively. The rebleeding rate was statistically significant between the two groups $(P=0.007)$. Variceal obliteration was achieved in one session in all patients in group I, whereas a few patients in group II required two sessions to achieve complete obliteration $(P<0.001$, $>$ Table 2$)$.

MRI confirmed hemiplegia of the left limb due to an embolism in the right middle cerebral artery after N-butyl-2-cyanoacrylate injection in one patient in group II. Other complications such as fever, chest pain, bleeding from post-treatment variceal ulcer, spontaneous bacterial peritonitis, hepatic encephalopathy, and mortality were not statistically different between the groups $(P>0.05$, $>$ Table 2$)$.

- Fig. 1 depicts representative images of a cirrhotic patient with a history of gastric variceal bleeding after endoscopic clipping prior to GVO treatment. - Fig. 2 shows representative images of N-butyl-2-cyanoacrylate injection alone.

\section{Discussion}

According to Sarin classification, IGV1 has the highest rate of bleeding (78\%) and mortality compared to the other three types [3]. Management of IGV1 is relatively difficult as there is no consensus regarding optimum treatment. The most com- 

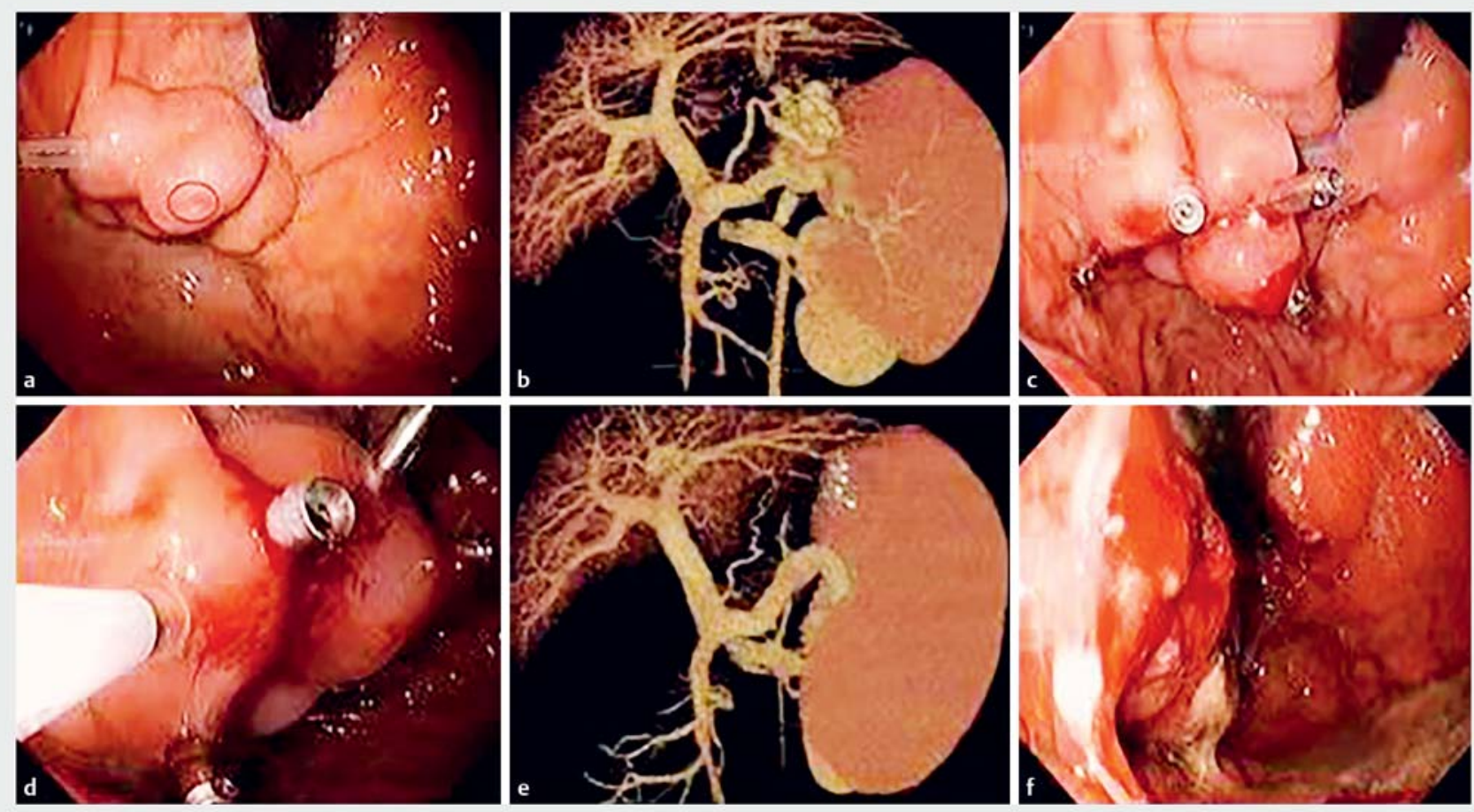

- Fig. 1 a On endoscopy, a hemorrhagic spot was seen in the huge gastric fundal varix. b Computed tomography (CT) scan showing the gastric varix with a large gastrorenal shunt. $\mathbf{c}$ Four clips were deployed on the varix. $\mathbf{d}$ "Modified Sandwich method" was used to complete the procedure. e Follow-up CT scan 3 weeks later showed the blocked gastric varix and gastrorenal shunt. $\mathbf{f}$ View during follow-up endoscopy 1 month later showing an injected ulcer and obliterated varix.

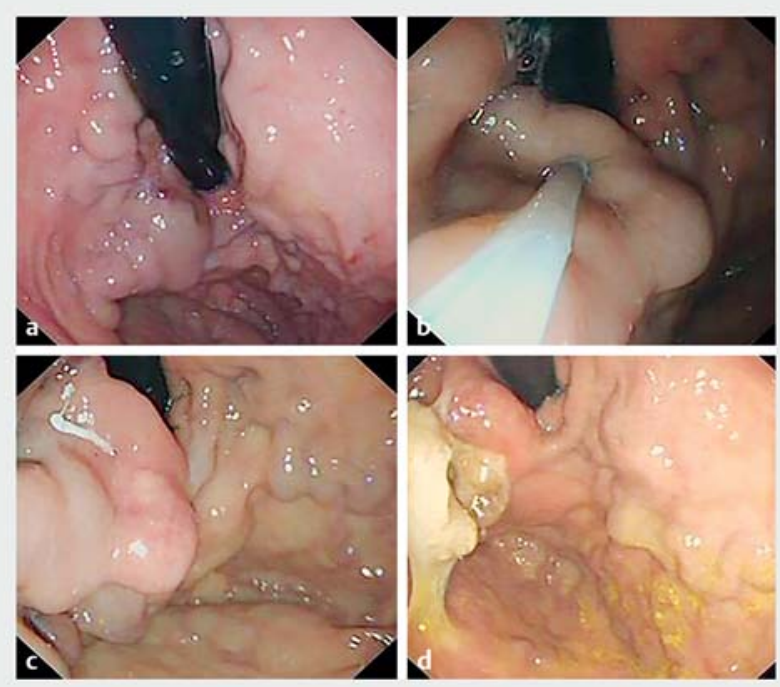

- Fig. 2 a A gastric fundal varix was visible on endoscopy. b "Modified Sandwich method" was used to complete the procedure. $\mathrm{c}$ Vessel has been solidified after two injections. d At 1-month follow-up, endoscopy showed well blocked GV.

monly used treatment is $\mathrm{N}$-butyl-2-cyanoacrylate injection, but there is a high risk of ectopic embolization, especially in patients with spontaneous GRS or SRS. Therefore, complete endoscopy and CTA scans are imperative to evaluate the status of spontaneous shunts, and for better treatment to avoid complications such as ectopic embolism.

Balloon-occluded retrograde transvenous obliteration (BRTO) and transjugular intrahepatic portosystemic shunt (TIPS) are second-line options in case of endoscopic treatment failures or alternatives for patients with large spontaneous shunts $[8,9]$. In recent years, endoscopic ultrasonography (EUS)-guided treatment of fundal varices with coil and/or N-butyl-2-cyanoacrylate reportedly has been highly effective in curbing active bleeding. Use of tissue adhesives in conjunction with endoscopic methods also is effective for post-GVO rebleeding and primary and secondary bleeding prophylaxis [10, 11]. Combination therapy thus appears to be safer and could mitigate risk of ectopic embolism.

Previous studies have shown that approximately $60 \%$ to $85 \%$ of GV patients have spontaneous shunts, particularly GRS and SRS $[12,13]$. Blood flow in the gastroesophageal junctional varices type is from the left gastric vein, which then enters the systemic circulation through the azygous vein. Blood flow of IGV1 is from the short gastric veins or posterior gastric vein, which then enters the inferior vena cava through the GRS/SRS shunt $(90.91 \%)$ [14]. In this study, CTA identified $89.39 \%$ patients with GRS (59/66), which was consistent with previous reports [14]. Although the "modified sandwich method" is effective in GV, ectopic embolism poses a serious problem, especially in IGV1 with large diameters, rich blood flow in the presence 
of spontaneous GRS or SRS. Arresting or reducing blood flow of GV prior to treatment can significantly reduce the possibility of ectopic embolization. For example, in the BRTO method, a balloon is used to block the blood flow through the GRS or SRS.

The clipping apparatus was originally developed to achieve endoscopic hemostasis of non-variceal gastrointestinal bleeding [15]. Endoscopic clipping was found to be as effective as band ligation, more so in treatment of EV [16]. Arantes [17] used titanium clips to successfully treat emergency GV bleeding, however, the patient was rehospitalized after 4 months with massive fundal variceal bleeding and had to undergo surgery. Morimot [18] reported a case of GV bleeding in a patient with alcoholic cirrhosis where BRTO treatment was repeated 16 days after hemostasis achieved with titanium clips. Perusal of the relevant literature thus revealed that titanium clips can block gastric variceal blood flow and arrest bleeding for only a short period of time. And, as they fall off by themselves within 1 to 2 weeks, hemostasis by titanium clip is a temporary event. Based on these findings, we designed a new method of endoscopic clipping prior to GVO for treatment of patients with IGV1 [19] and compared its efficacy and side effects with N-butyl-2-cyanoacrylate injection alone. We surmised that if titanium clips could block GV blood flow, GVO can be performed by endoscopists alone, which will reduce the complexity of the procedure.

In our study, we found that 46 patients with titanium clips did not have bleeding from gastric variceal injury. Even in patients with bleeding, titanium clips could stop the bleeding instantly. We also found that titanium clips significantly reduced the degree of GV, and most importantly, clipping prior to GVO provided better conditions for subsequent N-butyl-2-cyanoacrylate injections as the needle could be pulled out without any bleeding ensuing from the injection site.

All 96 patients achieved timely and successful treatment, especially for bleeding varices. The mean number of injection points per session and the dose of $\mathrm{N}$-butyl-2-cyanoacrylate

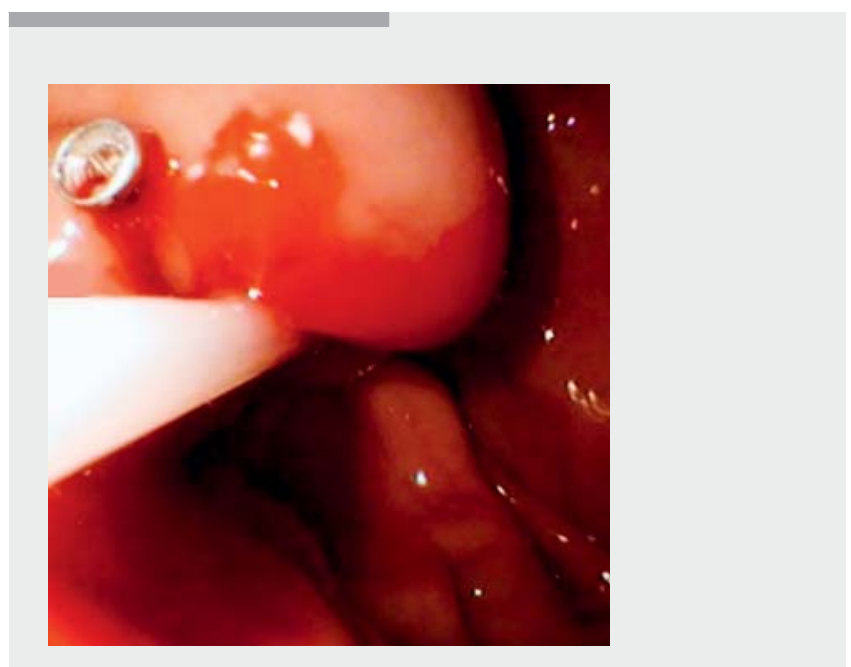

Video 1 A gastric varix with large gastrorenal shunt is treated endoscopically with deployment of two clips followed by injection of N-butyl-2-cyanoacrylate. were higher in group I compared with group II. The rebleeding rate of group I $(4.35 \%)$ was significantly lower than that of group II (18.00\%). Variceal obliteration was achieved in one session in all patients in group I while two sessions were required to achieve complete variceal obliteration in group II. This may be due to the decrease in variceal size and ease of obliteration after the first session of clipping.

Although rare, ectopic embolism such as cerebral embolism and pulmonary embolism is the most serious complication that can occur in patients receiving GVO [4]. Incidences of complications such as bleeding from postoperative ulcers, bacterial infections, fever, and embolism increase with the increase in the dose of N-butyl-2-cyanoacrylate [5]. In this study, only one patient in group II suffered from hemiplegia due to ectopic cerebral embolism. No embolism was recorded in group I. Studies have reported the rate of bleeding is approximately $6 \%$ after treatment with tissue adhesives [20]. However, we did not find postoperative bleeding in any of the 96 patients treated with $\mathrm{N}$ butyl-2-cyanoacrylate. By using a transparent injection needle, we made certain that the variceal blood returned to the needle, thereby ensuring that the N-butyl-2-cyanoacrylate was well flushed into the varices, and there was minimal submucosal damage and ulcerative hemorrhage.

\section{Conclusion}

In conclusion, results from this study confirm earlier reports that endoscopic clipping prior to GVO is safer for preventing ectopic embolization due IGV1 bleeding than traditional GVO. The results also showed that clipping before N-butyl-2-cyanoacrylate injection decreased the rebleeding rate and increased variceal obliteration significantly. However, validation of these findings requires a trial in a larger cohort and long-term clinical follow-up.

\section{Competing interests}

None

\section{References}

[1] Helmy A, Hayes PC. Review article: current endoscopic therapeutic options in the management of variceal bleeding. Aliment Pharmacol Ther 2001; 15: $575-594$

[2] Trudeau W, Prindiville T. Endoscopic injection sclerosis in bleeding gastric varices. Gastrointest Endosc 1986; 32: 264-268

[3] Sarin SK, Lahoti D, Saxena SP et al. Prevalence, classification and natural history of gastric varices: a long-term follow-up study in 568 portal hypertension patients[J]. Hepatology 1992; 16: 1343-1349

[4] Cheng LF, Wang ZQ, Li CZ et al. Low incidence of complications from endoscopic gastric variceal obturation with butyl cyanoacrylate. Clin Gastroenterol Hepatol 2010; 8: 760 - 766

[5] Marion-Audibert AM, Wallet F, Duperret S et al. Acute fatal pulmonary embolism during cyanoacrylate injection in gastric varices. Gastroenterol Clin Biol 2008; 32: $926-930$ 
[6] Hashizume M, Kitano S, Yamaga $\mathrm{H}$ et al. Endoscopic classification of gastric varices. Gastrointest Endosc 1990; 36: $276-280$

[7] Hou MC, Lin HC, Kuo Bl et al. Clinical implications of the white nipple sign and its role in the diagnosis of esophageal variceal hemorrhage. Am J Gastroenterol 1996; 91: 2103-2109

[8] Garcia-Tsao G, Bosch J. Management of varices and variceal hemorrhage in cirrhosis[J]. N Engl J Med 2010; 362: 823-832

[9] de Franchis R. Revising consensus in portal hypertension: report of the Baveno $V$ consensus workshop on methodology of diagnosis and therapy in portal ypertension[J]. ] Hepatol 2010; 53: 762-768

[10] Bhat YM, Weilert F, Fredrick RT et al. EUS-guided treatment of gastric fundal varices with combined injection of coils and cyanoacrylate glue: a large U.S. experience over 6 years (with video). Gastrointest Endosc 2016; 83: $1164-1172$

[11] Artifon ELA, Marson FP, Khan MA. Endoscopic ultrasonography-guided hemostasis techniques. Gastrointest Endosc Clin N Am 2017; 27: $741-747$

[12] Watanabe K, Kimura K, Matsutani S et al. Portal hemodynamics in patients with gastric varices. A study in 230 patients with esophageal and/or gastric varices using portal vein catheterization. Gastroenterology 1988; 95: 434-440
[13] Matsumoto A, Hamamoto N, Nomura T et al. Balloon-occluded retrograde transvenous obliteration of high risk gastric fundal varices. Am J Gastroenterol 1999; 94: 643-649

[14] Zhao LQ, He W, Ji M et al. 64-row multidetector computed tomography portal venography of gastric variceal collateral circulation. World J Gastroenterol 2010; 16: $1003-1007$

[15] Hayashi T, Yonezawa M, Kuwabara T. The study on stanch clip for the treatment by endoscopy. Gastroenterol Endosc 1975; 17: $92-101$

[16] Yol S, Belviranli M, Toprak S et al. Endoscopic clipping versus band ligation in the management of bleeding esophageal varices. Surg Endosc 2003; 17: 38-42

[17] Arantes V, Albuquerque W. Fundal variceal hemorrhage treated by endoscopic clip [J]. Gastrointest Endosc 2005; 61: 732

[18] Morimoto T, Fu KI, Konuma $\mathrm{H}$ et al. Endoscopic hemostasis with hemoclips for active gastric variceal bleeding[J]. Endoscopy 2010; 42: $333-334$

[19] Li HX, YE D, Kong DR. Endoscopic clipping prior to n-butyl-2-cyanoacrylate injection for gastric varices with a large gastrorenal shunt. Endoscopy 2018; 50: E102-E103

[20] Wang YM, Cheng LF, Li N et al. Study of glue extrusion after endoscopic N-butyl-2-cyanoacrylate injection on gastric variceal bleeding. World J Gastroenterol 2009; 15: 4945-4951 